\title{
The art of collecting experimental data internationally: EXFOR, CINDA and the NRDC network
}

\author{
H. Henriksson ${ }^{1, a}$, O. Schwerer ${ }^{2}$, D. Rochman ${ }^{3}$, M.V. Mikhaylyukova ${ }^{4}$, and N. Otuka ${ }^{5,6}$ \\ 1 OECD Nuclear Energy Agency (NEA) Data Bank, Issy-les-Moulineaux, France \\ 2 Nuclear Data Section, International Atomic Energy Agency (IAEA), Vienna, Austria \\ 3 National Nuclear Data Center, Brookhaven National Laboratory (BNL), Upton, NY, USA \\ 4 Nuclear Data Center (CJD), Institute of Physics and Power Engineering Institute (IPPE), Obninsk, Russian Federation \\ 5 Nuclear Reaction Data Center (JCPRG), Faculty of Science, Hokkaido University, Sapporo, Japan \\ 6 Nuclear Data Center, Japan Atomic Energy Agency (JAEA), Tokai, Japan
}

\begin{abstract}
The world-wide network of nuclear reaction data centres (NRDC) has, for about 40 years, provided data services to the scientific community. This network covers all types of nuclear reaction data, including neutroninduced, charged-particle-induced, and photonuclear data, used in a wide range of applications, such as fission reactors, accelerator driven systems, fusion facilities, nuclear medicine, materials analysis, environmental monitoring, and basic research. The now 13 nuclear data centres included in the NRDC are dividing the efforts of compilation and distribution for particular types of reactions and/or geographic regions all over the world. A central activity of the network is the collection and compilation of experimental nuclear reaction data and the related bibliographic information in the EXFOR and CINDA databases. Many of the individual data centres also distribute other types of nuclear data information, including evaluated data libraries, nuclear structure and decay data, and nuclear data reports. The network today ensures the world-wide transfer of information and coordinated evolution of an important source of nuclear data for current and future nuclear applications.
\end{abstract}

\section{Introduction}

The network of nuclear reaction data centres (NRDC) was established to collect experimental data from all over the world, mainly addressing data needs of the fission reactor industry. The present aim of the NRDC is to encompass all types of nuclear reaction data, including neutron- and chargedparticle-induced data, as well as photonuclear data, considering the requirements of many other nuclear applications, such as accelerator driven systems, fusion reactors, nuclear medicine, materials analysis, environmental monitoring, and basic research [1].

The four core nuclear data centres in the NRDC are the US National Nuclear Data Center (NNDC), the OECD Nuclear Energy Agency Data Bank (NEADB), IAEA Nuclear Data Section (NDS), and the Russia Nuclear Data Center (Centr Jadernykh Dannykh-CJD). The NEADB originates from the European Nuclear Energy Agency (ENEA) Neutron Data Compilation Centre (CCDN), established to collect neutron data, mainly from Europe and Japan, in a Neutron Data Direct Access System, "NEUDADA". The compiled data was transmitted to the BNL National Neutron Cross Section Center (NNCSC), which later was renamed to NNDC.

At the IAEA-NDS, the Data Storage and Retrieval System, "DASTAR" was produced for the storage and retrieval of neutron data. This DASTAR system was also used at the CJD centre to transmit nuclear data information between the IAEANDS and the CJD centre.

\footnotetext{
${ }^{a}$ Presenting author, e-mail: henriksson@nea.fr
}

The NRDC has evolved from these four centres, and include now nine additional specialized nuclear data centres based on particular types of reactions and/or geographic regions. The names and web site addresses of all the centres are listed in table 1.

Experimental data as well as theoretical models and evaluations are collected directly from the data producer or via a detailed scanning process of all available literature on nuclear data in the world. The search for data and scanning of articles, books, technical reports, doctoral theses and conference/workshop proceedings are coordinated by the NRDC, and discussed at annual meetings between all the participating centres. The first meeting of the four core nuclear data centres was held in 1966, and annual meetings have been organized since then.

The data is exchanged in a standardised format, so that the individual centres can employ their local databases. The consequent distribution of the data is finally carried out via search forms on the web retrieval pages (see table 1). The data is also distributed on CD/DVD for easy access together with software for data manipulation and display.

The NRDC network maintains the experimental database EXFOR [2] (also called CSISRS in the US [3]) and the bibliographical database CINDA [4]. EXFOR is the main source of experimental nuclear reaction data and the database is further described in detail below. CINDA has recently been extended to cover all references of the EXFOR database. The latest progress is described below. The individual nuclear data centres also distribute all types of nuclear data information, including evaluated data libraries, nuclear structure and decay data, and nuclear data reports. 
Table 1. Name and Internet address to the participating nuclear data centres in the NRDC network.

\begin{tabular}{|c|c|}
\hline Data Centre & Internet address \\
\hline $\begin{array}{l}\text { National Nuclear Data } \\
\text { Center, BNL, USA } \\
\text { OECD Nuclear Energy } \\
\text { Agency Data Bank, France }\end{array}$ & www.nea.fr/html/databank \\
\hline $\begin{array}{l}\text { IAEA Nuclear Data } \\
\text { Section, Austria }\end{array}$ & $\begin{array}{l}\text { www-nds.iaea.org } \\
\text { Mirror sites: } \\
\frac{\text { www-nds.ipen.br }}{\text { www-nds.indcentre.org.in }}\end{array}$ \\
\hline $\begin{array}{l}\text { Russian Nuclear Data } \\
\text { Center (CJD), Russia }\end{array}$ & www.ippe.ru/podr/cjd \\
\hline $\begin{array}{l}\text { Russian Nuclear Structure } \\
\text { and Reaction Data Center } \\
\text { (CAJAD), Russia }\end{array}$ & www.kiae.ru \\
\hline $\begin{array}{l}\text { Centre for Photonuclear } \\
\text { Experiments Data, Russia }\end{array}$ & cdfe.sinp.msu.ru \\
\hline $\begin{array}{l}\text { Chinese Nuclear Data } \\
\text { Center (CNDC), China }\end{array}$ & $\begin{array}{l}\text { http://159.226.2.40/ } \\
\text { (in Chinese) }\end{array}$ \\
\hline $\begin{array}{l}\text { JAEA Nuclear Data } \\
\text { Center, Japan }\end{array}$ & wwwndc.tokai-sc.jaea.go.jp \\
\hline $\begin{array}{l}\text { Nuclear Reaction Data } \\
\text { Center (JCPRG), Japan }\end{array}$ & www.jcprg.org/ \\
\hline $\begin{array}{l}\text { ATOMKI Charged- } \\
\text { Particle Nuclear Reaction } \\
\text { Data Group, Hungary }\end{array}$ & www.atomki.hu \\
\hline $\begin{array}{l}\text { Nuclear Data Center } \\
\text { (UKRNDC), Ukraine }\end{array}$ & ukrndc.kinr.kiev.ua \\
\hline $\begin{array}{l}\text { Center of Nuclear Physics } \\
\text { Data (CNPD), Russia }\end{array}$ & www.vniief.ru \\
\hline $\begin{array}{l}\text { KAERI Nuclear Data } \\
\text { Evaluation Lab., Korea }\end{array}$ & atom.kaeri.re.kr \\
\hline
\end{tabular}

\section{The NRDC network objectives}

The objectives of the NRDC network include the compilation of experimental nuclear data and bibliographic information, collection and promotion of evaluated general and special purpose data, exchange of nuclear data of all types with other data centres, development of common formats for computerised exchange of nuclear data, co-ordinated development of computer software for managing and disseminating nuclear data and documentation of current and future data needs in order to be able to meet changing user demands. Applications where nuclear data serves are many. The main use is for the $\mathrm{R} \& \mathrm{D}$ in nuclear energy, both concerning fission and fusion, but accelerator design, shielding, nuclear waste, safeguards, medicine, environmental monitoring, materials analysis, dosimetry, radiation safety and nuclear astrophysics are also implicated.

\section{The databases EXFOR and CINDA}

One of the main tasks of the NRDC network is to maintain, develop and promote the experimental database EXFOR and the bibliographical database CINDA. EXFOR is the main source of experimental nuclear reaction data and covers results published as early as in the 1930s. CINDA has recently been upgraded to contain charged-particle data, apart from neutroninduced and photo-induced data previously.

\subsection{CINDA}

Herbert Goldstein developed in the 1950s a scheme for keeping track of bibliographic neutron cross-section measurements, both concerning published and unpublished literature. This became later on the "Computer Index for Neutron Data" [3].

Today, the database CINDA (Comprehensive Index of Nuclear Reaction Data) contains bibliographical references to measurements, calculations, reviews and evaluations of cross-sections and other microscopic neutron-, gamma- and charged-particle data as well as references to computer libraries of numerical data. An automated preparation of CINDA from EXFOR was performed so that CINDA now covers all references in EXFOR. Over 180,000 lines were added from EXFOR, and the complete CINDA database contains now over 435,000 lines of bibliographic information, including 55,000 publications.

The CINDA database is organised in such a way that data is grouped by target nuclide, reaction/quantity and laboratory. All entries for a particular experiment are grouped together in one "block", a feature that considerably facilitates the search for experimental data and distinguishes CINDA from all other bibliographies. The database master file is maintained at the IAEA/NDS, where all the automated preparation of CINDA from EXFOR is taking place.

CINDA can be retrieved on web from most of the data centres in the NRDC network, it can be requested on CD-ROM or DVD, and the entire bibliography is also available as an archival book (CINDA 2006) in 7 volumes [4].

\subsection{EXFOR}

EXFOR was originally conceived for the exchange of neutron data and it was accepted as the official exchange format of the core data centres in 1969, designed for flexibility in order to meet the diverse needs of the NRDC. The NEADB (ENEACCDN at that time) and the IAEA-NDS wrote translation codes to convert their DASTAR and NEUDATA files, so that EXFOR would cover all these data. EXFOR is now the most important database of experimental reaction data in the world, with over 16,000 experiments, and about 110,000 tables of data. 


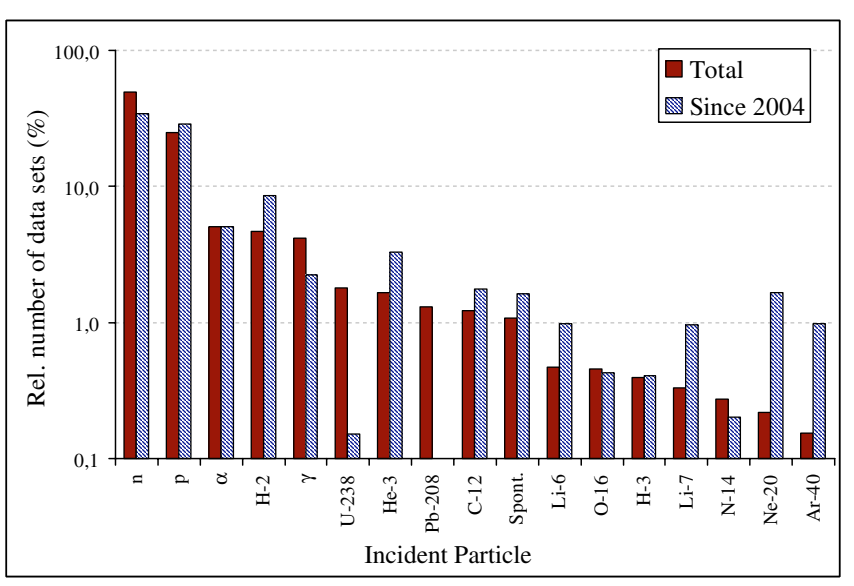

Fig. 1. The relative number of data sets in EXFOR plotted versus incident particle (note, the logarithmic scale). The two data sets represent the situation in EXFOR in total, and for references published since 2004.

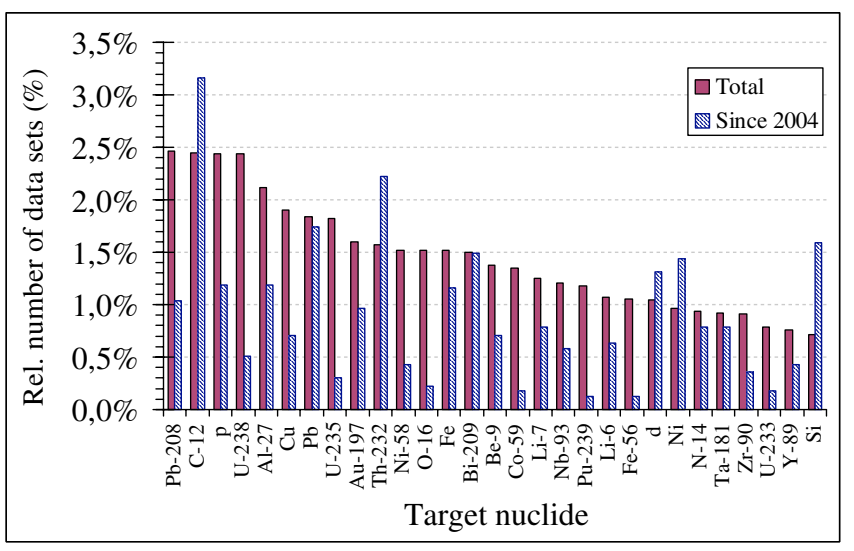

Fig. 2. The relative number of data sets for a certain target in EXFOR, in total and data published since 2004. The order of the isotopes is on decreasing total importance in EXFOR, e.g., there are in total most data sets with the target ${ }^{208} \mathrm{~Pb}$ (over 3,000 data sets).

As a result of two meetings held in 1975 and 1976 the format was further developed and adapted to cover all nuclear reaction data [2], which enlarged the NRDC network to encompass additional specialized nuclear data centres for compilation of particular types of reactions.

\subsection{Compilation efforts}

EXFOR includes integral, differential and partial cross sections of many types including angular distributions and Legendre coefficients, secondary particle spectra, polarization data, resonance parameters, fission product yields, nu-bar, thick target yields and product yields, reaction rates, resonance integrals, and many others.

The compulsory compilation of data comprises reactions with incident particles up to $\mathrm{A}=12$ of energies up to $1 \mathrm{GeV}$. Other data (heavier projectiles, higher energies and other more exotic data) are also compiled on a voluntary basis. Figure 1 shows recent data additions to EXFOR as a function of incident particle. It is shown that light charged particle induced data increase faster than the neutron-induced data in recent years. Figure 2 shows what target nuclides have been used in the experiments included in EXFOR. The graph points at the fact that most recent experiments are performed on ${ }^{12} \mathrm{C}$, ${ }^{232} \mathrm{Th}, \mathrm{d}, \mathrm{Ni}$ and Si targets. The order of the targets in the graph reflects the most measured targets in EXFOR in total (e.g., ${ }^{208} \mathrm{~Pb}$ is the most common target nuclide).

\subsection{Collection of data}

The most important source of experimental data is through direct contact with the data producer. Often, the data is well described, and tables are published or made available to the NRDC timely in connection to the publishing of the work. But, in many cases, data is only available in graphs (in particular for old articles where no author can be traced anymore) and has to be extracted manually. There are therefore a few scanning tools available in the NRDC network to digitize the data as well as possible. One example is the recently upgraded Japanese code GSYS-2 [5]. Programs to facilitate the compilation with specific EXFOR editors are also developed within the NRDC network.

Experimental data as well as theoretical models and evaluations are collected from all available literature on nuclear data in the world. The search for data and scanning of articles, books, technical reports, doctoral theses and conference/workshop proceedings are coordinated by the NRDC, and discussed at annual meetings between all the participating centres. The current status of recent compilations is updated weekly so that all data centres can follow what publications are being prepared.

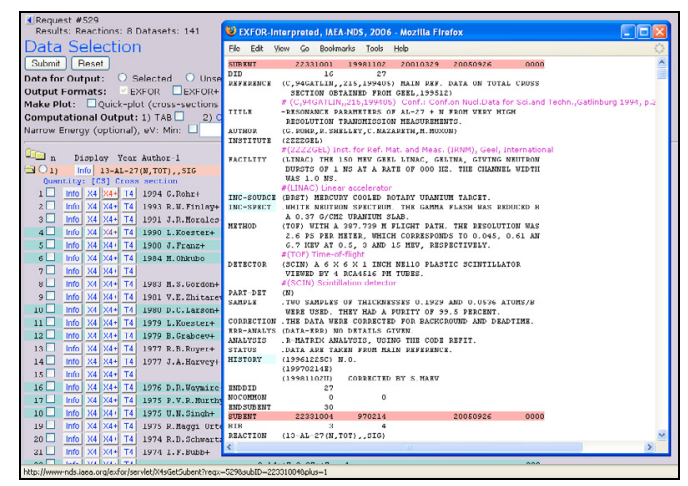

Fig. 3. The "X4+" format from the IAEA-NDS Web site [6].

\subsection{EXFOR end-user formats}

The EXFOR format is designed for the exchange of data within the NRDC. Several end-user formats for processing, comparing and plotting data have recently been improved. Two examples of user-friendly formats are shown in figures 3 and 4. The "X4+" or "EXFOR interpreted" format from the IAEA-NDS (fig. 3) [6] and the Janis EXFOR output format (fig. 4) [7] give the user explanations of the internal codes and abbreviations used. Computational end-user formats, which can include tabular data of one specific reaction/quantity from various measurements, are also available, such as "C4". 


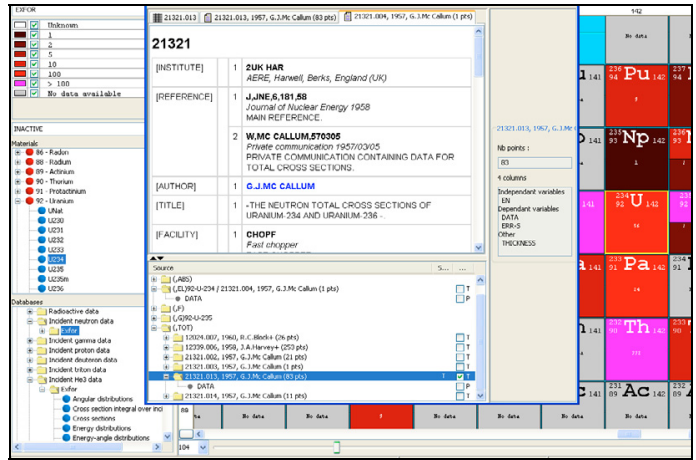

Fig. 4. The EXFOR output format as viewed in the data display program Janis [7].

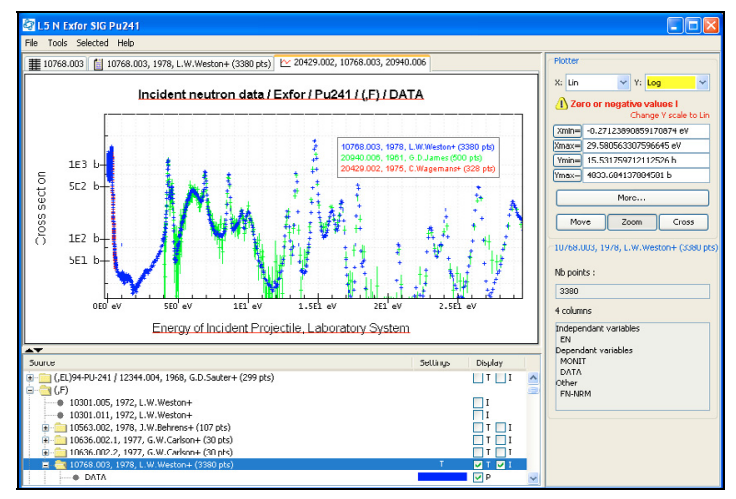

Fig. 5. EXFOR data plotted in Janis-3 [7].

\section{Services provided}

Services provided by the centres, mostly free of charge, consist of database retrievals on the web pages, experimental and evaluated data, distribution of nuclear data and computer programs on CD-ROMs/DVDs, as well as preparation and publication of reports and handbooks. Databases are continuously upgraded to new software platforms to facilitate access, retrieval and plotting [6-9]. An example of EXFOR data is plotted in figure 5 with the NEADB display program Janis.

\section{Evaluated data}

The NRDC services cover also evaluated data libraries, as they are important for users of nuclear data. References to the most common libraries are included in CINDA. The major general purpose libraries, ENDF/B-VII.0 (USA), JEFF-3.1 (NEA), BROND2.2 (Russia), JENDL-3.3 (Japan), CENDL-2.1 (China) and several specialised libraries, such as FENDL-2.1, are available online from NEADB (database "EVA") [9], NNDC and NDS (database "ENDF"). Evaluated and experimental data can be plotted online, or by using the software packages ZVView (downloaded from NDS or NNDC) [6] or JANIS (downloaded from NEADB) [7].

\section{Challenges and future developments}

The EXFOR database has recently been inter-compared and a common EXFOR master file was established. This common file is now maintained at the NDS in Vienna, where each centre can access it. There is an emphasis on adding important old data for particular applications (such as medical, astrophysics, ion beam analysis), and better completeness in photonuclear data is also envisaged. Increased emphasis is put also on quality control, in particular to corrections of mistakes in old compilations.

Other nuclear data-related activities specific to individual centres are also considered, such as the production of nuclear data handbooks and the creation of specific efforts within the NEA working party on International Nuclear Data Evaluation Cooperation (WPEC) subgroup activities, and the IAEA Coordinated Research Projects (CRPs). Among the present WPEC subgroups can be mentioned the high priority nuclear data request list [10], and the nuclear data needs for advanced reactor systems [11].

\section{References}

1. V.G. Pronyaev, O. Schwerer, The Nuclear Reaction Data Centres Network, Report INDC(NDS)-401 Rev. 4 (August 2003).

2. V. McLane, EXFOR Basics. A short guide to the nuclear reaction data format, Report BNL-NCS-63380 (IAEA-NDS-206), May 2000 (revision 2007 in preparation).

3. N.E. Holden, A short history of CSISRS, At the Cutting Edge of Nuclear Data Information Storage and Retrieval Systems and its Relationship to CINDA, EXFOR and ENDF, BNL-752882005-IR.

4. CINDA Archive 2006, The comprehensive Index to Nuclear Reaction Data (1935-2006), OECD Nuclear Energy Agency (2007, in preparation).

5. A. Minoguchi, K. Arai, N. Otuka, K. Naito, GSYS: Development and usage of a software to read-in and digitize the graphical data, INDC(JPN)-194/U, p. 19, http://www.jcprg.org/gsys/.

6. V. Zerkin, A. Trkov, Development of IAEA nuclear reaction databases and services (these proceedings).

7. N. Soppera et al., Recent updates to the nuclear data tool Janis (these proceedings), http://www.nea.fr/janis/.

8. B. Pritychenko et al., Nuclear Reaction and Structure Databases of the National Nuclear Data Center, $12^{\text {th }}$ Int. Symp. on Capture Gamma-ray Spectroscopy and Related Topics, Notre Dame, USA, 4-9 Sept. (2005), p. 589.

9. A. Hasegawa et al., Nuclear data activities at the NEA Data Bank (these proceedings).

10. A. Plompen et al., The NEA High Priority Nuclear Data Request List for future needs (these proceedings).

11. M. Salvatores, Nuclear data needs for advanced reactor systems, A NEA Nuclear Science Committee initiative (these proceedings). 